\title{
PROJETO AXIOMÁTICO COMO UM PROBLEMA DE PROGRAMAÇÃO MATEMÁTICA
}

\author{
Sdepan Bogosian Neto \\ Centro de Projetos de Navios - Marinha do Brasil \\ Ilha das Cobras, Ed. 16, s/no. Centro, Rio de Janeiro CEP 20091-000 \\ e-mail: sbogosian@gmail.com
}

\begin{abstract}
RESUMO
Este artigo apresenta a metodologia (ou teoria) do Projeto Axiomático aplicada ao projeto do flutuador de uma chata. Apresenta o entendimento de que o Projeto Axiomático seja parcela de um problema de programação matemática, que, assim como outras metodologias, consegue reduzir o número de interações necessárias ao balanceamento do projeto e atingir, numa certa vizinhança, a solução ótima. Enfim, tece considerações acerca da aplicabilidade do Projeto Axiomático ao Projeto de Navios.

Palavras-Chave: Projeto Axiomático; Projeto de Navios; Programação Matemática; Flutuador; Chata.
\end{abstract}

\begin{abstract}
This article presents the methodology (or theory) of the Axiomatic Design (AD) applied to the design of a barge float. It presents the idea that the Axiomatic Design is part of a Mathematical Programming problem. The article also shows that AD can, as well as other methodologies, reduce the number of required interactions to balance the ship design and it can also achieve, inside a neighborhood, the optimal solution. Finally it presents some considerations about the applicability of Axiomatic Design to ship design.
\end{abstract}

Keywords: Axiomatic Design; Ship Design; Mathematical Programming; Float; Barge.

\section{Latinos}

\section{LISTA DE SÍMBOLOS}

A - Matriz de Projeto - Design Matrix.

$A_{f}$ - Área total de cada flutuador.

$B$ - Boca da Chata.

$b$ - Boca do flutuador.

$D P$ - Design parameters (parâmetros de projeto).

$D P_{i}$ - Design parameter de ordem seqüencial i.

Dwt - Porte Bruto - Deadweight - diferença entre o deslocamento leve e o carregado.

$h$ - calado do flutuador e da Chata .

$L$ - Comprimento da Chata.

$l$ - Comprimento do Flutuador.

$P_{c}$ - Peso da Chata.

$P V_{i}-$ Variável do processo de fabricação $i$.

$V_{\text {sub }}$ - Volume Submerso dos Flutuadores e da Chata.

FR - Funcional requirements (requisitos de funcionalidade do objeto - requisitos do cliente). 
QFD - Quality Function Deployment.

Gregos

$\gamma_{\text {estr }}$ - peso específico da estrutura

$\gamma_{a g u a}$ - peso específico da água

\section{INTRODUÇÃO}

O projeto de engenharia de embarcações (projeto de navios) é um método para se obter, como produto final, o conceito e, quando em fases mais avançadas, toda documentação necessária à construção de um navio.

O projeto de navios é um problema que, entre suas muitas peculiaridades, não oferece, em seu estágio inicial, todas as informações necessárias à sua solução; mas estas informações, paulatinamente, são obtidas a partir do desenvolvimento das atividades de projeto.

Muitas destas atividades, entretanto, interagem (PIERONI E NAVEIRO, 2006) necessitando de dados de outras que lhe são feitas em sequência, o que requer serem refeitas em grau de detalhamento maior (ou seja, feitas com maiores informações) até que haja um balanceamento da solução, que muitas vezes pode não ser boa, em face de que a busca feita conduziu a um ponto balanceado; mas que não seja ótimo.

Como a quantidade de atividades é muito grande é facilmente possível que a disposição de atividades de projeto o conduza a interações desnecessárias. Neste sentido, surgem métodos como a Design Structure Matrix (PIERONI E NAVEIRO, 2006) cuja função é organizar o processo de projeto e com isso reduzir o número de interaçôes.

Quando se conseguem obter equações matemáticas capazes de aglutinar quantidade suficiente de variáveis desconhecidas, a programação matemática naturalmente reduz a interatividade e conduz à solução ótima. Este uso é comum nos centros mais avançados de engenharia (um exemplo é a Universidade de São Paulo, BRINATI, AUGUSTO, CONTI; 2007).

Outra forma de projetar é o Projeto Axiomático que também depende de equações, incorporando a redução do nível de interatividade e a condução a uma solução ótima.

Neste sentido, escreveu-se o presente artigo que mostra que o Projeto Axiomático pode ser entendido como parcela de um programa de programação linear e o aplica à criação de um flutuador para uma chata.

Destaque-se porém que não houve uma escolha a priori do método, mas ocorreu em função do amadurecimento do conhecimento sobre o projeto em questão.

$\mathrm{O}$ projeto do flutuador a que se referia este artigo era grandemente interativo, isto porque o peso do flutuador interferia no peso da estrutura total que interferia no calado, que deveria permanecer constante. Por outro lado, modificações nas formas do flutuador faziam-no modificar seu peso e resistência estrutural.

Neste sentido, optou-se por criar um modelo matemático que diminuísse interatividade do processo.

Ao se perceber a semelhança que existia entre o modelo criado e a prática do projeto axiomático, percebeu-se que a forma de desenvolvimento do projeto se incluía perfeitamente na classificação de projeto axiomático. Como até o momento não se conhecia uma aplicação na Engenharia Naval para esta metodologia de projeto, resolveu-se escrever este artigo.

Optou-se, porém, por uma descrição simplificada do problema em que tanto flutuador como chata são considerados elementos em forma de paralelepípedo, de modo a preservar os dados sem prejuízo do propósito do artigo. 


\section{O PROJETO AXIOMÁTICO}

O projeto axiomático, ou design axiomático, é uma tentativa de matematicamente equacionar as necessidades do usuário no objeto a ser projetado (FRs - functional requirements) aos parâmetros físicos deste projeto - (DP - design parameters), por meio de uma matriz que os relaciona, a "matriz de projeto" $\left[A_{i j}\right]$. Este tipo de definição lembra bastante a "casa da qualidade" do método QFD, com a diferença de que, no método QFD, a "matriz de relações" (ESTORILIO, 2007, p. 19) que relaciona os "requisitos do cliente" às "características do produto" é composta qualitativamente.

Portanto, o projeto axiomático pode ser escrito numa única equação:

$$
\begin{aligned}
& {\left[\begin{array}{l}
F R_{1} \\
F R_{2} \\
\cdots \\
F R_{n}
\end{array}\right]=\left[\begin{array}{cccc}
A_{11} & A_{12} & \ldots & A_{1 n} \\
A_{21} & \ldots & & \\
\ldots & & & \\
& & & \\
A_{n 1} & A_{n 2} & & A_{n n}
\end{array}\right]\left[\begin{array}{l}
D P_{1} \\
D P_{2} \\
\cdots \\
D P_{n}
\end{array}\right]} \\
& {\left[\begin{array}{l}
F R_{1} \\
F R_{2} \\
\cdots \\
F R_{n}
\end{array}\right]=\left[\begin{array}{cccc}
\frac{\partial F R_{1}}{\partial D P_{1}} & \frac{\partial F R_{1}}{\partial D P_{2}} & \cdots & \frac{\partial F R_{1}}{\partial D P_{n}} \\
\frac{\partial F R_{2}}{\partial D P_{1}} & \cdots & & \\
\cdots & & & \\
\frac{\partial F R_{n}}{\partial D P_{1}} & \frac{\partial F R_{n}}{\partial D P_{2}} & & \frac{\partial F R_{n}}{\partial D P_{n}}
\end{array}\right]\left[\begin{array}{l}
D P_{1} \\
D P_{2} \\
\cdots \\
D P_{n}
\end{array}\right]} \\
& \{F R\}=\left[A_{i j}\right]\{D P\}
\end{aligned}
$$

\section{Axiomatic Design Framework}

The Concept of Domains

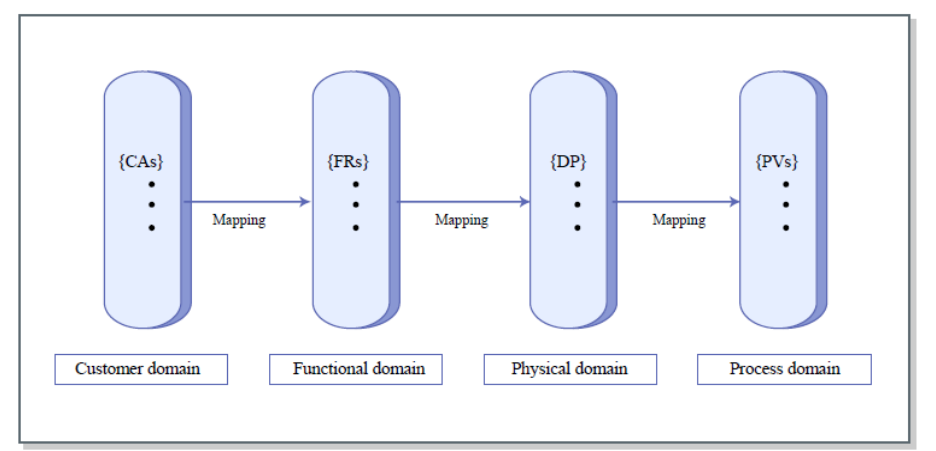

Figura 1 - Domínios do Projeto Axiomático. Fonte: Sun, Lee 2005.

Entretanto, o Projeto Axiomático também pode envolver outros domínios. Sun e Lee (2005) citam o domínio do consumidor, o domínio da funcionalidade, o domínio físico e o domínio do processo que aparecem na figura 1 - extraída da referência. Estes mesmos autores propõem uma matriz que traz alguns exemplos. No caso de materiais, por exemplo, explicam que o domínio do consumidor é representado pelo desempenho desejado; no domínio funcional, as 
propriedades requeridas para um certo desempenho; no domínio físico, a microestrutura; e no domínio do processo, os processos para conseguir aquela microestrutura.

Deste modo, o projeto como um todo acresce à estrutura da matriz de projeto também outras matrizes, conforme a equação (iv), no caso do domínio do processo:

$$
\left[\begin{array}{l}
D P_{1} \\
D P_{2} \\
\cdots \\
D P_{n}
\end{array}\right]=\left[\begin{array}{cccc}
\frac{\partial D P_{1}}{\partial P V_{1}} & \frac{\partial D P_{1}}{\partial P V_{2}} & \cdots & \frac{\partial D P_{1}}{\partial P V_{n}} \\
\frac{\partial D P_{2}}{\partial P V_{1}} & \cdots & & \\
\cdots & & & \\
\frac{\partial D P_{n}}{\partial P V_{1}} & \frac{\partial D P_{n}}{\partial P V_{2}} & & \frac{\partial D P_{n}}{\partial P V_{n}}
\end{array}\right]\left[\begin{array}{l}
P V_{1} \\
P V_{2} \\
\cdots \\
P V_{n}
\end{array}\right]
$$

A base, segundo os autores e segundo Axiomatic... (1999) depende de dois axiomas:

Axioma 1: O Axioma da Independência. Os requisitos de funcionalidade (FR) são independentes. Axioma 2: O Axioma da Informação. O conteúdo de informação do projeto deve ser minimizado.

Assim, para aplicação do projeto axiomático, deve-se bem escolher os FR (requisitos do cliente) e as variáveis de que estes FRs dependem.

A vantagem é que a aplicação do projeto axiomático diminui o trabalho de projeto. Conforme seus autores, o projeto axiomático tem as seguintes vantagens (extrato de Suh, 2003):

1. Prover um meio sistemático de projetar produtos e grandes sistemas;

2. Tornar os projetistas humanos mais criativos;

3. Reduzir a busca aletória nos projetos;

4. Minimizar a iteratividade e o processo de tentativa e erro - usada no caso do projeto do flutuador;

5. Determinar as quais as melhores configurações para continuidade do projeto;

6. Criar arquitetura que capturam completamente a funcionalidade do sistema e provêem pronta documentação;

7. Dotar o computador de poder criativo.

\section{O PROJETO AXIOMÁTICO ENQUANTO PROJETO PARAMÉTRICO}

Projetos de navios que atendem a requisitos de um armador ou requisitos de um estado maior, quando navios de guerra, são balanceados, isto é, atendem a uma solução ótima global.

$\mathrm{O}$ projeto segue com atividades em seqüência "considerando as necessidades e requisitos do cliente, sendo conduzido até um nível de detalhamento que possibilite a elaboração de documentos, desenhos e uma especificação técnica que permitam a contratação de um estaleiro para a construção do navio" (EDAMATU, 2008, p. 10).

Diferentemente do projeto que segue em atividades seqüenciais, existe o projeto paramétrico que usa métodos de otimização para atingir a medida de mérito econômica (PARSONS, 2003) - o que matematicamente é uma função objetivo. O projeto paramétrico trabalha, então, relacionamentos entre variáveis de projeto (que correspondem aos DP do projeto axiomático) e não com valores constantes dos fatores de projeto, tentando com isso, tanto otimizar o tempo, como a qualidade do resultado final (ibid.).

A título de exemplo de projeto paramétrico típico, tem-se Vangbo $(2011$, p. 63) em que aparece um típico programa de programação linear que facilmente pode ser entendido como o acréscimo da matriz $\mathrm{A}$, do projeto axiomático, à critérios de otimização. A figura 2 é um extrato de Vangbo (2011, p. 63) em que aparecem a função objetivo multicritério $\mathrm{Z}$ e variáveis típicas de projeto de navio como a altura metacêntrica $(\mathrm{KM})$ e o volume submerso $(\nabla)$. 


$$
\begin{array}{ll}
\operatorname{Min} Z=0.8 C_{w_{-} a w l 1}+0.2 C_{w_{-} d w l 2}+0.8 S_{d w l 1}+0.2 S_{d w l 2} \\
0.2 \leq p_{z 7} \leq 0.9 & {[-]} \\
0.2 \leq p_{y 6} \leq 0.9 & {[-]} \\
\nabla \geq 20000 & {\left[\mathrm{~m}^{3}\right]} \\
K M_{d w l 1} \geq \frac{D}{2} & {[\mathrm{~m}]} \\
K M_{d w l 2} \geq \frac{D}{2} & {[\mathrm{~m}]} \\
\left\{\text { Start: } p_{z 7}=0.9\right. & \left.p_{y 6}=0.9\right\}
\end{array}
$$

Figura 2- Otimização de um casco por Vangbo (2011, p. 63)

No ponto de vista do autor do presente artigo, o projeto axiomático é uma espécie de projeto paramétrico em que a otimização acontece, sim, mas em torno de uma "vizinhança" de um ponto multidimensional do projeto, posto que os requisitos de projeto FRs são satisfeitos por meio de uma matriz A, que é tanto mais distante dos pontos de projeto quanto maior o afastamento das derivadas parciais da hipersuperfície em que se encontra o ponto de projeto viável.

\section{CÁlCULO DO FLUTUADOR}

Arbitraram-se as seguintes hipóteses simplificadoras:

(h.1) O peso do flutuador seria sua área construída pela densidade de área de estrutura (já reforçada). A densidade de área da estrutura seria calculada com base no peso de uma seção de uma chapa com reforços;

(h.2) O calado do flutuador é o mesmo da chata;

(h.3) O calado é dado;

(h.4) O peso da Chata é dado e vale $P_{c}$;

(h.5) O Deadweight (Porte Bruto, $D w t$ ) é um parâmetro requerido, ou seja, é dado;

(h.6) O flutuador terá um pontal ligeiramente maior que o calado de modo a não ser totalmente submerso, em águas calmas, de um valor $a$;

(h.7) utilizou-se o equilíbrio entre peso e volume para se diminuir os cálculos matriciais;

(h.8) o valor do comprimento do flutuador foi arbitrado o mesmo comprimento da chata, ou seja, $L=l$;

(h.9) os flutuadores são simétricos e anexados aos bordos da chata.

Partiu-se, então, ao projeto do flutuador, lembrando-se que o peso do flutuador interfere no peso da estrutura total que interfere no calado, que, neste projeto, deve permanecer constante. $\mathrm{O}$ modo de se trabalhar foi criar uma função analítica que incorporasse o peso do conjunto e permitisse igualar o peso ao empuxo (volume submerso), equação (11).

\subsubsection{Desenho do Dispositivo}

A figura 2 apresenta o desenho do dispositivo. 

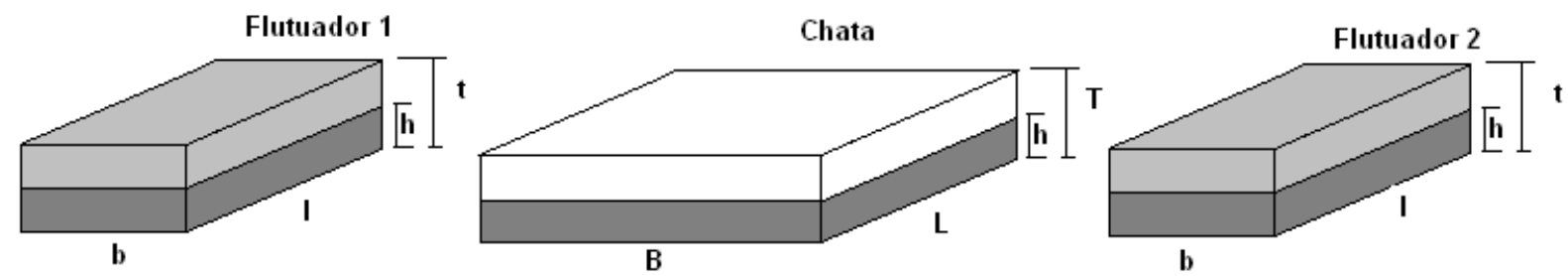

Figura 2 - Desenho do Dispositivo Chata-Flutuadores

\subsubsection{Volume Submerso}

\subsection{3 Área Total de cada Flutuador}

$$
V_{s u b}=h[B L+2 b l]
$$

$$
\begin{aligned}
& t=a+h \\
& A_{f}=2[b l+t b+t l] \\
& A_{f}=2 b[t+l]+2 t l
\end{aligned}
$$

\subsubsection{Peso total}

$$
P_{T}=2 A_{f} \gamma_{e s t r}+P_{c}+D w t
$$

\subsubsection{Equilíbrio entre peso e volume}

$$
\begin{aligned}
& P_{T}=h(B L+2 b l) \gamma_{\text {agua }}=h B L \gamma_{\text {agua }}+2 b l \gamma_{\text {agua }} \\
& P_{T}=2[2 b(t+l)+2 t l] \gamma_{\text {estr }}+P_{c}+D w t
\end{aligned}
$$

Igualando as expressões (6) e (7):

$$
\begin{gathered}
h B L \gamma_{\text {agua }}+2 b l \gamma_{\text {agua }}=2[2 b(t+l)+2 t l] \gamma_{\text {estr }}+P_{c}+D w t \\
2 b\left[l \gamma_{\text {agua }}-2(t+l)\right] \gamma_{\text {estr }}=4 t l \gamma_{\text {estr }}+P_{c}+D w t+h B L \gamma_{\text {agua }} \\
b=\frac{4 t l \gamma_{\text {estr }}+P_{c}+D w t+h B L \gamma_{\text {água }}}{2\left[l \gamma_{\text {agua }}-2(t+l)\right] \gamma_{\text {estr }}}
\end{gathered}
$$

\subsubsection{FR; DP e Matriz de Projeto}

$$
b=\left[\begin{array}{lll}
\frac{4 t \gamma_{\text {estr }} h B L \gamma_{\text {agua }}}{2\left[l \gamma_{\text {agua }}-2(t+l)\right] \gamma_{\text {estr }}} & 1 & 1
\end{array}\right]\left[\begin{array}{l}
1 \\
D w t \\
P_{c}
\end{array}\right]
$$

A equação (11) apresenta a solução do final da boca , $b$, que permite o dimensionamento do flutuador da chata, onde o comprimento da chata é o mesmo que do flutuador e o calado também já foi definido.

Como se pode perceber, todos os elementos da matriz $A$ são constantes e o único $F R$ é a boca do flutuador. Na verdade, a Matriz $A$ degenerou-se num vetor.

\section{CONSIDERAÇÕES A CERCA DE APLICABILIDADE AO PROJETO DE NAVIO}

O presente cálculo nada mais foi que o cálculo do equilíbrio entre peso e empuxo necessários ao dimensionamento da boca do flutuador. Disso decorreu a simplicidade da matriz de projeto, $A$, e foi consequência do emprego das hipóteses (h.1) a (h.9) em que todos os demais fatores foram considerados constantes. 
O conteúdo que deve ter o projeto de um navio pode ser encontrado na item 0313 da NORMAN 01 em que é estabelecido para obtenção da Licença de Construção (LCEC) das Embarcações "SOLAS" . Nele em que fica clara a necessidade de muitas outras tarefas, ou seja, documentos, de modo a se obter a licença de construção. A Tabela 1 apresenta a documentação requerida ao projeto do navio e a comparação ao que se fez no presente emprego do projeto axiomático.

Tabela 1 - Relação de Tarefas/Documentos de um projeto de navio

\begin{tabular}{|c|c|}
\hline 0313 da NORMÁN 01 & Requisito Cumprido em um Projeto Axiomático \\
\hline 1) Memorial Descritivo & $\begin{array}{l}\text { Aspecto legal - item descritivo - não se aplica ao } \\
\text { projeto, mas sim é uma espécie de registro. }\end{array}$ \\
\hline 2) Plano de Linhas; & $\begin{array}{l}\text { Pela sua simplificação (paralelepípedo) não foi } \\
\text { necessário, entretanto, é requerido para o cálculo do } \\
\text { volume submerso. }\end{array}$ \\
\hline 3) Plano de Arranjo Geral; & $\begin{array}{l}\text { Pela sua simplificação, o desenho 3D esquemático } \\
\text { serviu às finalidades requeridas, entretanto, seria } \\
\text { necessário para identificar pontos de reboque, etc. }\end{array}$ \\
\hline 4) Curvas Hidrostáticas e Cruzadas; & $\begin{array}{l}\text { Necessárias para um projeto axiomático de um } \\
\text { navio/flutuador que não fosse um paralelepípedo. }\end{array}$ \\
\hline 5) Plano de Capacidade; & $\begin{array}{l}\text { Informação não relevante para a tarefa de cálculo de } \\
\text { equilíbrio. Por outro, lado os volumes dos tanques } \\
\text { seriam necessários a um projeto axiomático. }\end{array}$ \\
\hline $\begin{array}{l}\text { 6) Plano de Arranjo de Luzes de } \\
\text { Navegação; }\end{array}$ & $\begin{array}{l}\text { Não se incorporou esta informação e não é requisito } \\
\text { necessário ao projeto axiomático. }\end{array}$ \\
\hline 7) Plano de Seção Mestra; & Pré-requisito para o cálculo do peso estrutural \\
\hline 8) Plano de Perfil Estrutural; & Não é requerido \\
\hline 9) Plano de Expansão do Chapeamento; & Não é requerido \\
\hline 10) Plano de Segurança & Não é requerido \\
\hline 11) Plano de Combate a Incêndio; & Não é requerido \\
\hline 12) Plano de Revestimentos; & Não é requerido \\
\hline 13) Arranjo de Forros e Anteparas; & Não é requerido \\
\hline 14) Relatório da Prova de Inclinação; & Só pode ser concluída após a chata ser lançada. \\
\hline $\begin{array}{l}\text { 15) Folheto de Trim e Estabilidade } \\
\text { Intacta }\end{array}$ & Necessária para o conjunto do flutuador \\
\hline 16) Manual de Carregamento de Grãos; & Necessária para o conjunto do flutuador \\
\hline $\begin{array}{l}\text { 17) Folheto de Trim e Estabilidade em } \\
\text { Avaria, em duas vias; }\end{array}$ & Necessária para o conjunto do flutuador \\
\hline $\begin{array}{l}\text { 18) Plano de Emergência para Prevenção } \\
\text { da Poluição por Óleo (SOPEP) }\end{array}$ & Necessária para o conjunto do flutuador \\
\hline $\begin{array}{l}\text { 19) Manual de Peiação de Carga (Cargo } \\
\text { Securing Manual), em duas vias. }\end{array}$ & Necessária para o conjunto do flutuador \\
\hline $\begin{array}{l}\text { 20) Anotação de Responsabilidade } \\
\text { Técnica (ART) referente ao projeto de }\end{array}$ & Aspecto legal. \\
\hline 21) CTS Provisório. & Aspecto legal. \\
\hline
\end{tabular}

Uma análise criteriosa dos documentos requeridos para projeto permite perceber que para um projeto axiomático, os documentos 4) e 7) são importantíssimos, entretanto, neste exercício simplificado, não foram requeridos, porque se tratava de um navio paralelepipedal. 


\section{CONCLUSÕES}

O projeto do flutuador é grandemente interativo, porque o peso do flutuador interfere no peso da estrutura total que interfere no calado, que, neste projeto, deve permanecer constante. $\mathrm{O}$ modo de se trabalhar foi criar uma função analítica que incorporasse o peso do conjunto e permitisse igualar o peso ao empuxo (volume submerso).

Existe aplicabilidade do projeto axiomático ao projeto do navio, entendido, como se fez aqui, como uma modalidade de projeto paramétrico, com a consequiência esperada de diminuição da iteratividade do projeto.

Enfim, o projeto axiomático também concorre para a otimização do projeto, mas em torno de uma vizinhança do ponto de projeto, pela natureza da matriz de projeto, $A$, uma matriz de derivadas parciais.

\section{REFERÊNCIAS BIBLIOGRÁFICAS}

BRASIL - Ministério da Defesa - Marinha do Brasil - Diretoria de Portos e Costas. "Normas da Autoridade Marítima para Embarcações Empregadas na Navegação de Mar Aberto". Edição 2005.

BRINATI, H. L., AUGUSTO, O. B., CONTI, M. B. de "Learning Aspects of Procedures for Ship Conceptual Design Based on First Principles." International Conference on Engineering Education - ICEE 2007, Coimbra, 2007, Sept. 3 - 7.

EDAMATU, Flavio Jun. "Novos Processos de Construção Naval e seus reflexos sobre a competitividade da indústria naval brasileira: O ressurgimento da Indústria Naval brasileira e a adoção pela Marinha dos novos métodos de Projeto e Construção Naval”. Monografia apresentada à Escola de Guerra Naval, 2008.

ESTORILIO, Carla "QFD: Desdobramento da Função Qualidade". Apostila do Departamento Acadêmico de Mecânica da Universidade Tecnológica Federal do Paraná, Curitiba-PR, 2007. Disponível em: http://pessoal.utfpr.edu.br/vrubel/arquivos/_1_PI-2_QFD-apostila.pdf Acesso: 22 nov. 2013.

PARSONS, M., G., "Parametric Design, in Ship Design and Construction", Edited by Lamb, T., New Jersey, The Society of Naval Architecture and Marine Engineers, 2003, pp 11-1 to 11-48.

PAHL, G., BEITZ, W., FELDHSEN, J., GROTE, K. H. "Konstruktionslehre - Methoden und Anwendug", 6th Ed., Tradução para o português de Hans Andreas Werner. São Paulo: Edgard Blücher, 2005.

PIERONI, E.; NAVEIRO, R. M. "Integrating Project Management, Concurrent Engineering, and Engineering Design to Improve Ship Design". Third International Conference on Production Research - Americas' Region 2006 Americas' Region 2006 Region 2006 (ICPR----AM06) Disponível em: http:// www.produtronica.pucpr.br/icpr-am06 / Accepted\%20 for \%20 Oral\%20 Presentation/ 5. \%20 Product\%20 Engineering/Paper\% 20127.pdf Acesso: 14 jun. 2014

SUN, N.; "Complexity: Theory and Applications". Disponível em: moodle.stoa.usp.br/mod/resource/view.php?id, 2003. Acesso: 22 nov. 2013. 
SUN, N.; LEE, T. "2.882:System Design and Analysis based on AD and Complexity Theories". [s.1.]: [s.n.], 2005. Disponível em: http://ocw.mit.edu/courses/mechanical-engineering/2-882system-design-and-analysis-based-on-ad-and-complexity-theories-spring-2005/lecturenotes/lec202.pdf Acesso: 22 nov. 2013.

VANGBO, Olav Petter "CFD in Conceptual Ship Design". Dissertação de Mestrado da Norwegian University of Science and Technology - NTNU, Trodheim, 2011. 